\title{
Mis6/CENP-I maintains CENP-A nucleosomes against centromeric non-coding transcription during mitosis
}

\author{
Hayato Hirai ${ }^{1}$, Yuki Shogaki ${ }^{1}$ and Masamitsu Sato ${ }^{1,2,3, *}$ \\ 1 Laboratory of Cytoskeletal Logistics, Department of Life Science and Medical \\ Bioscience, Graduate School of Advanced Science and Engineering, \\ 2 Institute for Medical-oriented Structural Biology, \\ ${ }^{3}$ Institute for Advanced Research of Biosystem Dynamics, \\ Waseda Research Institute for Science and Engineering, Waseda University, TWIns, 2- \\ 2 Wakamatsucho, Shinjuku-ku, Tokyo 162-8480, Japan. \\ *To whom correspondence should be addressed: masasato@waseda.jp
}

\section{Summary}

Centromeres are established by nucleosomes containing the histone H3 variant CENP-A. CENP-A is recruited to centromeres by the Mis18-HJURP machinery. During mitosis, CENP-A recruitment ceases, implying the necessity of CENP-A maintenance at centromeres, although the exact underlying mechanism remains elusive. Herein, we show that the kinetochore protein Mis6 (CENP-I) retains CENPA during mitosis in fission yeast. Eliminating Mis6 during mitosis caused immediate loss of pre-existing CENP-A at centromeres. CENP-A loss occurred due to the transcriptional upregulation of non-coding RNAs at the central core region of centromeres, as confirmed by the observation RNA polymerase II inhibition preventing CENP-A loss from centromeres in the mis 6 mutant. Thus, we concluded that Mis6 blocks the indiscriminate transcription of non-coding RNAs at the core centromere, thereby retaining the epigenetic inheritance of CENP-A during mitosis. 


\section{Introduction}

Duplicated chromosomes must be equally distributed between daughter cells so that genetic information is adequately propagated to the progeny. The mitotic spindle attaches to the kinetochore, which is a macromolecular protein complex formed at the centromere, in order to equally separate sister chromatids to opposite spindle poles ${ }^{1}$.

In most eukaryotes, kinetochore assembly requires the deposition of CENP-A (Cnp1 in the fission yeast Schizosaccharomyces pombe), a centromere-specific variant of histone H3. In budding yeast, the centromere is composed of a 125-bp DNA sequence (called a point centromere), and a single histone octamer containing CENP-A is allocated to the centromere in addition to the usual H3-containing nucleosomes ${ }^{2}$. In contrast, multiple CENP-A-containing nucleosomes are deposited to the regional centromere, which consists of a 35 110-kb central core region flanked by pericentric regions in fission yeast $^{3}$, or megabases of repetitive DNA sequences in higher eukaryotes, including humans ${ }^{4}$.

Reduction of CENP-A levels at the centromere causes errors in chromosome segregation, which may result in aneuploidy, leading to cell death and birth defects ${ }^{5}$. Thus, the amount of CENP-A at the centromere is strictly regulated. Upon DNA replication, the number of nucleosomes containing CENP-A at the centromere is halved ${ }^{6}$, necessitating the replenishment of new CENP-A nucleosomes during the cell cycle especially prior to mitosis onset in order to assemble functional kinetochores. The timing of CENP-A supply differs between organisms, occurring in the early G1 phase in humans as opposed to mainly in $\mathrm{G} 2$ in fission yeast ${ }^{6,7}$.

Recruitment of CENP-A requires the Mis18 complex (Mis18BP1, Mis18a and Mis18 $\beta$ in humans ${ }^{8}$; Mis16, Mis18 and Mis19 (also known as Eic1 or Kis1) in fission yeast $^{9-12}$ ) as well as a chaperone HJURP (Holliday junction recognition protein; Scm3 in fission yeast) ${ }^{13-16}$. In budding yeast ${ }^{17}$, human cells ${ }^{18}$ and Drosophila cells ${ }^{19}$, histone $\mathrm{H} 3$ within euchromatin undergoes dynamic turnover during the cell cycle, while CENP-A nucleosomes are generally immobile. Once recruited to centromeres, they appear neither disassembled nor replaced by new nucleosomes containing CENP-A or H3 during both mitotic cycles and meiosis in higher eukaryotes ${ }^{6,20}$, implying the existence of machinery for CENP-A maintenance.

In various organisms, non-coding RNAs (ncRNAs) are transcribed at the central core region of centromeres ${ }^{21}$. When RNA polymerase II (RNAPII) progresses to the central core region of centromeric DNA to transcribe ncRNAs, nucleosomes are temporarily evicted from the DNA. Transcription-coupled turnover of CENP-A has also 
been reported when transcription of the alphoid array is artificially enhanced by tethering the herpes virus VP16 activation domain to a human artificial chromosome (HAC) ${ }^{22}$. Furthermore, S. pombe Cnp1 (CENP-A) dispersed when ncRNA transcription at the central core region was upregulated in cells lacking Mediator complex subunit Med2023. These findings indicate that CENP-A needs to be maintained against the transcription of centromeric ncRNAs, even outside DNA replication.

While CENP-A maintenance at centromeres is necessary during the cell cycle, the underlying molecular mechanism remains elusive. Ubiquitylation of CENP-A was recently shown to contribute to CENP-A maintenance in human cells ${ }^{24}$. Further, human kinetochore proteins CENP-C and CENP-N directly interact with CENP-A-containing nucleosomes in vitro ${ }^{25,26}$. These interactions are required for the immobility of CENP-Acontaining nucleosomes in human cells ${ }^{27,28}$. Another study suggested that CENP-C and CENP-N do not contribute to CENP-A maintenance in human cells ${ }^{29}$. Taken together, whether these factors are required for CENP-A retention in vivo remains controversial. It was recently demonstrated that HJURP is necessary for CENP-A maintenance during DNA replication ${ }^{30}$. However, HJURP dissociates from centromeres in metaphase both in human cells $8,13,14$ and in fission yeast ${ }^{9,15,16}$, indicating that HJURP does not engage in CENP-A maintenance during metaphase. It was recently reported that the histone chaperone Spt6, which is known as a histone H3 recycler, also contributes to the recycling of pre-existing CENP-A during ncRNA transcription at centromeres in both Drosophila and human cells ${ }^{31}$. However, whether more factors are involved in CENP-A maintenance remains unclear.

In this study, we demonstrate that the kinetochore protein Mis6 (CENP-I in human), which has been originally defined as a loading factor for CENP-A during interphase, contributes to maintenance of CENP-A during metaphase. We propose that Mis6 may counteract progression of RNAPII into core centromeres to prevent the reduction of CENP-A nucleosomes during metaphase. 


\section{Results}

\section{Cnp1 is not recruited to centromeres during metaphase}

The Mis18 complex and Scm3 (HJURP) are loading factors for Cnp1 (CENP-A) conserved in fission yeast and higher eukaryotes, such as chickens and humans ${ }^{8,9,13-16}$. In fission yeast, the kinetochore protein Mis6 (CENP-I) is considered another loading factor, as Cnp1 localisation is reduced in the mis6-302 temperature-sensitive mutant ${ }^{32}$. The Mis18 complex and Scm3 localise to kinetochores during interphase but are dispersed during mitosis, whereas Mis6 remains throughout the cell cycle ${ }^{9,15,16,33}$. This suggests that Cnp1 could be recruited at any time, assuming that Mis 6 would constantly serve as a Cnp1 loading factor.

To test this possibility, we examined the kinetics of Cnp1 localisation to centromeres using Cnp1-GFP (green fluorescent protein was tagged to the C-terminus of Cnp1) strains. Cnp1-GFP cells were arrested at G1, G2 and metaphase via cdc10-27, $c d c 25-22^{34}$ and alp12-182835 mutations, respectively. We then performed fluorescence recovery after photobleaching (FRAP) assays, where the Cnp1-GFP fluorescence intensity at centromeres was measured every 5 min after laser irradiation of the Cnp1GFP foci. We found that Cnp1-GFP fluorescence intensity gradually recovered after irradiation in G1- or G2-arrested cells (Fig. 1a-d), indicating that bleached Cnp1 was replaced, albeit slowly. In contrast, Cnp1-GFP intensity did not recover after irradiation in metaphase-arrested cells (Fig. 1e, f). These results indicate that $\mathrm{Cnp1}$ is not recruited to centromeres during metaphase, and Mis6 does not play a role in Cnp1 loading in the meantime, whereas Mis6 does contribute to Cnp1 recruitment during interphase by allocating $\mathrm{Scm} 3$ to centromeres ${ }^{16}$.

\section{Mis6 is required to maintain Cnp1 at centromeres during metaphase}

We speculated that Mis6 may play another role at the centromeres during metaphase. We first examined whether the function of Mis6 during metaphase is essential for viability. The mis6-302 mutant was combined with the cut9-665 mutant, which has defects in cell cycle transition from metaphase to anaphase because of the low APC/C (anaphase promoting complex/cyclosome) activity at restrictive temperature ${ }^{36}$. The resultant mis6-302 cut9-665 double mutant exhibited severe growth defects, even at semi-restrictive temperature (Fig. 2a). This suggests that the mitotic function of Mis6 is crucial for prolonged metaphase.

We then tried to determine the function of Mis6 during metaphase. As overexpression of Cnp1 suppressed the temperature sensitivity of the mis6-302 mutant, 
albeit partially ${ }^{9,37}$, the mitotic function of Mis6 would also be exerted for positive regulation of Cnp1. We then predicted that Mis6 plays a role in the maintenance of Cnp1 during metaphase.

To test this hypothesis, we performed assays to monitor Cnp1 maintenance during metaphase: WT (mis6 $6^{+}$) and mis6-302 cells expressing GFP-Cnp1 were synchronously arrested in metaphase in order to follow the kinetics of Cnp1 localisation to centromeres during metaphase. As illustrated in Fig. 2b, cells were first arrested in the $\mathrm{G} 1 / \mathrm{S}$ phase using hydroxyurea $(\mathrm{HU})$, followed by a temperature shift up to $36^{\circ} \mathrm{C}$ in order to inactivate Mis6 before mitotic entry. After HU washout, cells were released into mitosis until telophase (mitotic exit).

In WT cells, the intensity of a single GFP-Cnp1 dot in telophase was approximately half that in the previous G1/S stage. This reduction in GFP-Cnp1 intensity simply reflects the segregation of sister chromatids in the meantime. Therefore, Cnp1 was rarely removed from the centromeres during WT mitosis. This result is consistent with previous observations in human cells showing that pre-existing CENP-A at centromeres is retained throughout the cell cycle ${ }^{6}$. In the mis6-302 mutant, GFP-Cnp1 intensity during telophase was approximately $35 \%$ of that in G1/S (Fig. 2c), indicating that Cnp1 was dissociated from centromeres when cells passed through mitosis in the absence of Mis6. Thus, Mis6 is required for Cnp1 retention during mitosis.

It could be hypothesised that Scm3 (HJURP), rather than Mis6, is responsible for Cnp1 maintenance, because Scm3 and Mis6 are interdependent for localisation to centromeres ${ }^{15}$. This is unlikely, as Scm 3 ceases localisation to centromeres in WT cells during mitosis ${ }^{15,16}$. To further clarify this point, we isolated a temperature-sensitive scm3 mutant, in which the localisation of Mis6-GFP was unaffected at restrictive temperature (Supplementary Fig. S1 a-c). The mutant harboured a substitution at position 55 $\left(s c m 3^{S 55 P}\right)$, which was close to that in the previous $s c m 3^{L 56 F}$ mutant that also retained Mis6 localisation to centromeres ${ }^{16}$. In the $s c m 3^{555 P}$ mutant, the reduction in GFP-Cnp1 intensity after mitosis was almost comparable to that in WT cells ( 60\%, Fig. 2c), indicating that $\mathrm{Cnp} 1$ did not dissociate from centromeres during metaphase. Thus, Scm3 was dispensable for the maintenance of mitotic Cnp1.

To further investigate whether Mis6 retains Cnp1 during metaphase, WT and mis6-302 cells expressing GFP-Cnp1 were prepared similarly as in Fig. $\mathbf{2 b}$, but arrested to mitosis (metaphase) for $\geq 1 \mathrm{~h}$ by double inhibition using the microtubule poison carbendazim (CBZ) in combination with the a-tubulin temperature-sensitive mutant alp12-182835 (Fig. 2d). Metaphase arrest of the cell was confirmed by observing the Plo1 (Polo-like kinase) foci at spindle pole bodies ${ }^{38,39}$. The GFP-Cnp1 intensity at centromeres 
decayed more rapidly in mis6-302 cells than in WT cells (Fig. 2e, f). Taken together, these results suggest that Mis6, but not $\mathrm{Scm} 3$, is responsible for the maintenance of Cnp1 at centromeres during metaphase.

We examined whether the role of Cnp1 maintenance at mitotic centromeres is specifically assigned to Mis6 or shared with other kinetochore components by repeating similar GFP-Cnp1 maintenance assays using the mis12-537 and nuf2-2 mutants. These harbour mutations in Mis12 (belonging to the Mis12/Mtw1 subcomplex) and Nuf2 (to the Ndc80 subcomplex), respectively ${ }^{40,41}$. These components localised to the outer regions of centromeres relative to Mis6, and Mis6 localised to centromeres in the mis12 and nuf2 mutants (Supplementary Fig. S1d) ${ }^{41,42}$.

In both mutants, signal intensities of Cnp1 at centromeres during mitotic arrest were retained as in the WT and unlike in the mis6-302 mutant (Fig. $\mathbf{2 g}, \mathbf{~ h}$ ). These assays indicated that involvement in Cnp1 maintenance is not a common feature among kinetochore factors. Rather, it is specifically assigned to Mis6.

\section{Enforced dismissal of Mis6 during metaphase reduces Cnp1}

To further examine the function of Mis6 during mitosis, we sought to introduce experimental conditions in which Mis6 remains functional during interphase but is rapidly inactivated upon entry into mitosis. We developed a strategy for knocksideways experiments using Mis6-GFP and Kis1-GBP (GFP-binding protein ${ }^{43}$ ), as illustrated in Fig. 3a. Kis1 (Mis19 or Eic1) together with Mis16 and Mis18 forms the Mis18 complex to recruit Cnp1 to centromeres during interphase, whereas the whole complex disperses during metaphase $\mathrm{e}^{9-12}$ (top, Fig. 3a). Taking advantage of the turnover, the fusion protein Kis1-GBP may dismount Mis6-GFP from centromeres upon mitotic entry (bottom, Fig. 3a). As shown in Fig. 3b, Mis6-GFP and Kis1-GBP-mCherry co-localised to centromeres during interphase. In metaphase cells, the Mis6-GFP signal at centromeres substantially decreased, indicating that Mis6-GFP was removed from centromeres specifically during mitosis by the knocksideways system, as expected. Later in anaphase, Mis6-GFP and Kis1-GBP-mCherry re-localised together at centromeres.

We then evaluated whether Cnp1 localisation is reduced in response to the enforced removal of Mis6 during metaphase. In control cells (mis6-GFP kis 1 $^{+}$, Fig. 3c), mCherry-Cnp1 foci at centromeres were constantly detected along the spindle during prometaphase, and only $8 \%$ of control cells failed to retain mCherry-Cnp1 at centromeres (Fig. 3d). In contrast, in mis6-GFP kis1-GBP double-tagged cells, mCherryCnp1 signals were clearly detected at centromeres during the initial stage of mitosis $(0$ 
min, Fig. 3c and Supplementary Fig. S2), verifying that co-expression of Mis6-GFP and Kis1-GBP did not affect their functions in Cnp1 deposition during interphase. In contrast, immediately after mitosis onset, mCherry-Cnp1 signals dispersed from centromeres in $\sim 25 \%$ of the cells (Fig. 3c, d). Moreover, the double-tagged strain displayed growth defects at high temperature, further supporting the importance of Mis6 localisation during mitosis (Fig. 3e).

\section{Reduction of Cnp1 during metaphase is coupled to transcription at centromeres}

A major reason for Cnp1 loss during mitosis may be the transcription of ncRNAs in centromeres. ncRNAs are transcribed in various organisms, including plants, fission yeast and humans ${ }^{21}$. As our results indicate that Mis6 retains Cnp1 to centromeres, we focused on the relationship between ncRNA transcription at the central core region of centromeres and Cnp1 maintenance. The central core region (cnt) of centromeres is transcriptionally silenced in WT cells, whereas silencing is impaired in the mis6-302 mutant ${ }^{37}$. This was reproduced in our experiments using the cnt1::ura4+ strain, in which the $u r a 4^{+}$reporter gene conferring the uracil autotroph was inserted in the central core region of chromosome 1 (Fig. 4a). Growth of WT cells harbouring the cnt1::ura4+ insertion was defective in medium lacking uracil (SD-Ura) and was fine in the counterselection medium containing 5-fluoroorotic acid (FOA). This indicated that transcription in the central core region is subtle and is mostly silenced. In contrast, the mis6-302 mutant containing cnt1::ura4+ showed the opposite growth pattern, confirming that the central core region in the mis6-302 mutant was no longer silenced (Fig. 4a).

Likewise, mis12-537 and nuf2-2 mutants were used in centromeric silencing assays. In contrast to the mis6-302 mutant, both mis12-537 and nuf2-2 mutants showed restricted growth in the medium lacking uracil and retained their growth capacity in the presence of FOA at semi-restrictive temperatures (Supplementary Fig. S3a). These results demonstrate that kinetochore mutants with intact Mis6 localisation retained the ability to silence transcription at the central core region.

Chromatin IP experiments using an antibody against the C-terminalphosphorylated form (pSer5) of RNA polymerase II (RNAPII) detected enrichment of the central core sequences in the mis6-302 mutant compared to WT cells (Fig. 4b). In contrast, the enrichment was undetectable in both the mis12-537 and nuf2-2 mutants (Supplementary Fig. S3b), wherein Mis6 remained at centromeres. This demonstrates that active RNAPII transcribes ncRNA in the central core region when Mis6 is absent.

In general, conventional histone octamers containing $\mathrm{H} 3$ are temporarily dismantled by the chromatin remodelling factor Fun30 ( $\mathrm{Fft} 3$ in fission yeast), allowing 
RNAPII to proceed with transcription ${ }^{44-46 .}$

Analogously, Cnp1-containing octamers must be temporarily dissociated from centromeres upon transcription, which could be a potential reason for Cnp1 loss in mitotic mis6-302 cells. It is possible that $\mathrm{Fft} 3$ also removes Cnp1-containing octamers upon ncRNA transcription at the centromeres. When we followed the temporal kinetics of GFP-Cnp1 intensity during metaphase, the reduction of GFP-Cnp1 intensity seen in mis6-302 cells was cancelled by the additional knockout of Fft3 (mis6-302 fft3 Supplementary Fig. S4a), confirming that Fft3 removes Cnp1 upon transcription at centromeres.

To find further evidence of transcription-coupled Cnp1 dismantling, we tested whether Cnp1 loss still occurs when transcription is blocked by RNAPII inhibitors such as 1,10-phenanthroline and thiolutin. Experiments were designed as shown in Fig. 2d. In brief, the cells were arrested to metaphase using CBZ as well as through alp12-1828 mutation, and GFP-Cnp1 intensity was monitored in the presence or absence of RNAPII inhibitors (Fig. 4c). In WT cells, RNAPII inhibitor 1,10-phenanthroline did not affect the amount of Cnp1 at centromeres (WT, Fig. 4d, e). In mis6-302 cells without RNAPII inhibitor treatment, the GFP-Cnp1 intensity constantly decreased throughout metaphase, as shown in Fig. 2e, f. In contrast, 1,10-phenanthroline treatment resulted in similar GFPCnp1 intensity as in WT cells (Fig. 4d, e). Comparable results were obtained in assays using thiolutin as the RNAPII inhibitor (Supplementary Fig. S4b, c). Taken together, the reduction of GFP-Cnp1 during mitosis of the mis6-302 mutant is coupled to the transcription of ncRNAs at the central core region.

These results demonstrated that Mis6 prevents unnecessary transcription of ncRNAs at the central core region, thereby maintaining Cnp1 on chromatin during metaphase.

\section{Chromatin remodelling factor Spt6 is required for Cnp1 recycling during mitosis}

Transcription of ncRNAs in the central core region occurs at a certain level even in the presence of functional Mis6, as a minor amount of phosphorylated RNAPII was detected in the central core region (see Fig. 4b). It has been previously shown that phosphorylated RNAPII at the central core region is upregulated during the G2-M transition ${ }^{47}$. Thus, some RNAPII passes through the central core region due to the removal of Cnp1 by Fft3 in WT cells, while the Cnp1 fluorescence signal remained during metaphase (Figs. 2, 3). These results imply that Cnp1 is maintained through additional mechanisms, other than the Mis6-dependent system, during ncRNA transcription. Possible candidates include chromatin remodelling factors, such as the histone 
chaperone FACT (FAcilitates Chromatin Transcription) or Spt6, considered to act as recycling factors for nucleosomes after the passage of RNAPII at coding regions in the euchromatin ${ }^{44,45}$. Drosophila and human Spt6 have been shown to contribute to CENPA maintenance during interphase ${ }^{31}$. Thus, we tested whether these factors also contribute to Cnp1 recycling when central core ncRNAs are transcribed.

First, we determined the level of GFP-Cnp1 intensity over time during metaphase in the knockout mutant of Spt6 (spt6 $\Delta$ ) and the FACT component Pob3 (pob3 $\Delta$ ). Cells were arrested to metaphase using CBZ as well as through the alp12-1828 mutation (Fig. 5a). GFP-Cnp1 intensity decreased over time in spt6 $\Delta$ cells but not in pob3 $\Delta$ cells. The temporal kinetics of GFP-Cnp1 levels in pob3 $\Delta$ spt $6 \Delta$ double-knockout cells was similar to that in spt6 $\Delta$ cells (Fig. 5b and Supplementary Fig. S4d). This finding demonstrated that Spt6 recycles Cnp1 in WT cells, as a certain level of RNAPII leaks into the core centromere during mitosis.

During the interphase of Drosophila cells, HJURP deposits de novo CENP-A to centromeres, which is also maintained by $S p 6^{31}$. In contrast, de novo CENP-A deposition does not occur during metaphase, suggesting that the role of Spt6 in CENPA recycling may be particularly crucial in metaphase. Therefore, we investigated whether recycling Cnp1 by Spt6 during metaphase is crucial for chromosome segregation by assessing the segregation pattern of the cen2-GFP signal, with the centromeres of chromosome II visualised using GFP ${ }^{48}$. Cells were arrested to metaphase using the cut9665 mutant, followed by release into anaphase by re-activating Cut 9 function. As shown in Fig. 5c, deletion of Spt6 significantly reduced the number of cells showing equal segregation of cen2-GFP among cut9-665 cells.

In the absence of functional Mis6, a large amount of RNAPII appeared to surge into the central core region. A previous study indicated a direct interaction of Spt6 with RNAPII in human cells ${ }^{49}$. Therefore, we assessed whether there was an upregulation of Spt6 in the mis6-302 mutant. Chromatin IP assays using Spt6-GFP pull-downs demonstrated that DNA fragments corresponding to the central core region were not specifically enriched in the mis6-302 sample compared to the WT (mis6+) sample (Fig. 5d). As the amount of the histone recycler Spt6 at the central core region was unaffected despite RNAPII accumulation, Spt6 may not be able to sufficiently cope with the upsurge of ncRNA transcription occurring in the absence of Mis6.

This led us to postulate that two distinct mechanisms contribute to CENP-A maintenance during mitosis, that is, the Mis6-mediated system impedes the upsurge of RNAPII into the central core region, whereas Spt6 (FACT) recycles CENP-A in response to ncRNA transcription. 
To investigate this relationship, we compared the level of Cnp1 maintenance during metaphase of mis6-302 and spt6 $\Delta$ cells. Although both mutants were defective in Cnp1 maintenance during metaphase, mis6-302 cells showed more severe defects than spt6 $\Delta$ cells (Fig. 5e, f). Severe reduction of the GFP-Cnp1 signal at centromeres was also detected in the mis6-302 spt6 $\Delta$ double mutant to a similar degree as in mis6-302 (Fig. 5e, f). These results indicate that once Cnp1 is lost by an upsurge of ncRNA transcription in the mis6 mutant, the Spt6-mediated recycling system cannot fully operate for Cnp1 maintenance.

Taken together, we conclude that Mis6 is the primary factor that maintains Cnp1 during mitosis by blocking the invasion of RNAPII, which minimises the transcription of centromeric ncRNAs. The slight transcription leakage over the blockade causes the removal of Cnp1, which is then reintroduced via Spt6-mediated recycling.

\section{Discussion}

\section{The life cycle of CENP-A nucleosomes}

This study provides a new model for the temporal regulation of CENP-A nucleosomes during the cell cycle. That is, histone octamers containing CENP-A undergo cycles of deposition and turnover at the centromeric DNA. In fission yeast, CENP-A deposition is thought to occur during $S$ phase (upon DNA replication) as well as during the G2 phase ${ }^{7,50}$. Our FRAP analysis highlighted the incorporation of CENP-A into centromeres during the G1 phase (Fig. 1). After photobleaching, Cnp1-GFP intensity at interphase centromeres did not recover to its original state. Assuming that the turnover of pre-existing CENP-A or $\mathrm{H} 3$ from centromeres may be a prerequisite for the incorporation of new CENP-A, eviction may represent a rate-limiting step for subsequent CENP-A deposition in $S$. pombe.

We propose that CENP-A can be loaded onto centromeres at any time, except during mitosis (pro metaphase). This is in line with the behaviour of the Mis18 complex and Scm3 (HJURP) throughout the cell cycle, both dispersing from kinetochores during early mitosis but returning in late mitosis (anaphase) ${ }^{11,16}$. In contrast, the timing of deposition is limited to the early $\mathrm{G} 1$ phase in human cells $8,13,14$. This discrepancy may be related to differences in the regulation of cyclin-dependent kinase (CDK) activity in each organism.

In human cells, phosphorylation of the Mis18 complex and HJURP by CDK 
prevents their localisation to centromeres. Since multiple distinct CDKs operate, e.g., Cdk1/2-cyclin A (active from S/G2 phase until metaphase) and Cdk1/cyclin B (pro metaphase), CENP-A deposition by the Mis18 complex and HJURP is restricted only to the G1 phase, when they escape CDK phosphorylation ${ }^{51}$.

In fission yeast, a single CDK (Cdc2-Cdc13/cyclin B) may determine its substrates depending on the total level of activity ${ }^{52}$. It is possible that the Mis 18 complex and HJURP are phosphorylated only when CDK activity is substantially high, that is, during metaphase. This might explain why fission yeast cells are competent in CENP-A deposition at any time except metaphase. As deposition machinery is compromised during metaphase, cells employ specific mechanisms to not lose CENP-A nucleosomes meanwhile.

\section{The mechanism of CENP-A maintenance by CENP-I}

The present study elucidates a previously unexplored mechanism through which CENP-A is maintained at the central core region of centromeres. In particular, we demonstrated a new role for Mis6 (CENP-I) as a CENP-A maintenance factor. Mis6 has been considered a factor required for CENP-A deposition, since the localisation of Cnp1 and Scm3 is lost in the mis6 mutant ${ }^{15,32}$. However, we found that in the absence of functional Mis6, CENP-A at centromeres decreased during metaphase when CENP-A was no longer deposited. Therefore, this reduction directly reflects CENP-A turnover from the centromeres.

A recent study revealed that HJURP, in cooperation with the MCM complex, is required for CENP-A maintenance during $S$ phase in human cells ${ }^{30}$. However, we demonstrated that CENP-A was maintained from G1/S phase until the end of mitosis in the scm3 mutant (Fig. 2c). This suggests that Scm3 (HJURP) does not predominantly contribute to CENP-A maintenance in fission yeast. Instead, it is possible that Mis6 maintains CENP-A during interphase in addition to metaphase.

Transcription of centromeric ncRNA was previously detected throughout the cell cycle in S. pombe ${ }^{47}$, with only a certain fraction of RNAPII localising into the central core (cnt) region to transcribe ncRNAs ${ }^{23}$. As our results demonstrated that RNAPII binding to the $c n t$ region was elevated in the mis 6 mutant, we propose that Mis6 (CENP-I) can act as an insulator for RNAPII invasion into the central core region in other organisms as well. In human cells, unknown factors in addition to HJURP appear to maintain CENP. $A^{30}$. Moreover, ncRNA transcription at centromeres was detected in higher eukaryotes 
besides humans, including mice and the tammar wallaby ${ }^{21}$, suggesting that CENP-I possibly maintains CENP-A in these species as well. On the other hand, CENP-Ideficient chicken DT40 cells display stable localisation of CENP-A to centromeres ${ }^{53,54}$, suggesting that CENP-I might not contribute to CENP-A maintenance. Assuming that CENP-I serves as an insulator, it would be intriguing to investigate whether ncRNAs are actively transcribed at centromeres in DT40 cells. Molecular schemes for CENP-A maintenance may be intimately linked to whether ncRNA transcription occurs in centromeres. The utilisation of Mis6 (CENP-I) represents a suitable solution in organisms which actively transcribe centromeric ncRNAs, as it contributes to both CENP-A deposition and CENP-A maintenance as an RNAPII insulator.

\section{Two-step machinery for sustainable CENP-A positioning during mitosis}

Even though Mis6 impedes the progression of RNAPII into the cnt region of centromeres, transcripts are still detected at a certain level ${ }^{23}$. This indicates that CENP$\mathrm{A}$ in the $\mathrm{cnt}$ region is temporarily removed by the chromatin remodelling factor $\mathrm{Fft} 3$ when RNAPII proceeds but is mostly maintained by the histone recycler Spt6. Despite the accumulation of RNAPII within the cnt region of the mis 6 mutant, concomitant accumulation of the histone recycler Spt6 was not observed. Unlike in human cells ${ }^{49}, S$. pombe Spt6 might not directly interact with RNAPII. Alternatively, an intense upregulation of RNAPII in the cnt failed to efficiently recruit Spt6. In either case, the capacity of Spt6 at the central core region appears to be limited to recycling CENP-A only at basal levels.

We conclude that two mechanisms operate for CENP-A maintenance. First, Mis6 serves to insulate against the invasion of RNAPII into the core centromere, thus maintaining CENP-A, particularly during mitosis when de novo CENP-A deposition does not occur. Although Spt6 recycles CENP-A during mitosis in WT cells as the second machinery for CENP-A maintenance, the recycling capacity of Spt6 appears to be limited. Insulation of RNAPII by Mis 6 is thus employed as the primary machinery, followed by Spt6-mediated CENP-A recycling as a backup, in a stepwise strategy for the epigenetic maintenance of CENP-A until the next cell cycle.

\section{Acknowledgements}

We thank Y. Takayama, A. Yamamoto, Y. Hiraoka, T. Sakuno Y. Watanabe, M. Yanagida 
and the National Bioresource Project (NBRP) of Japan for the yeast strains. H.H. was a research fellow of the Japan Society for the Promotion of Science (JSPS; 16J09035). This study was supported by JSPS KAKENHI JP25291041, JP15H01359, JP16H04787, JP16H01317, JP18K19347 and 21H00261 to M.S. This study was also supported by The Uehara Memorial Foundation, Ohsumi Frontier Science Foundation and Waseda University grants for Special Research Projects 2017B-242, 2017B-243, 2018B-222, 2019C-570 and 2020R-038 to M.S. This work was also partly supported by the JSPS Core-to-Core Program, A: Advanced Research Networks.

\section{Author Contributions}

H.H. and M.S. conceived and designed the experiments. H.H. and Y.S. performed the experiments. H.H. wrote the draft of the manuscript. M.S. supervised the project and finalised the manuscript through discussion with H.H. and Y.S.

\section{Competing Interests}

The authors declare no competing interests. 


\section{Methods}

S. pombe strains and genetics Strains used in this study are listed in Supplementary Table S1. Standard PCR-based methods for gene targeting were employed for the construction of knock-out mutants and strains with fluorescent protein tagging ${ }^{55-57}$. We used multiple constructs for the visualisation of Cnp1. In Figure 1, strains expressing the Cnp1-GFP gene under an adh21 promoter at the $C$ locus (adjacent to the SPAC26F1.12c gene of chromosome 1) were used (the original strain was a gift from Y. Watanabe) ${ }^{58}$. In Fig. 2C, GFP-Cnp1 was expressed under the endogenous promoter (a gift from Y. Takayama) ${ }^{59}$. In other figures, the fusion gene of GFP-Cnp1 or mCherry-Cnp1 driven by the $n m t 1$ promoter was inserted at the CO2 locus of chromosome 2 (adjacent to the SPBPB7E8.01 gene) $)^{60}$ as an extra copy of the endogenous $c n p 1^{+}$gene.

To create these integrant strains, we first created plasmids harboured the $n m t 1$ promoter placed upstream of the cnp1 coding sequence fused with the GFP or mCherry gene via the Golden Gate method ${ }^{60}$. The constructed plasmids were digested by the Fsel enzyme for linearisation and introduced into strains to induce homologous recombination at the $\mathrm{CO} 2$ locus.

For knocksideways experiments, expression of Kis1-GBP and Kis1-GBPmCherry was induced via pREP1-based plasmids containing the nmt1 promoter. The GFP-kan gene was inserted to the end of the mis $6^{+}$coding sequence, so that the strain expresses the fusion protein of Mis6-GFP instead of the endogenous Mis6 protein.

Cells in Figs. 1, 2, 4, 5, S1, S3 and S4 were cultured in YE5S medium, while those in Figs. 3 and $\mathbf{S} 2$ were cultured in Edinburgh minimal medium (EMM).

Mutagenesis of the scm3gene We first constructed the scm3-myc-hph strain, in which the $\mathrm{scm}^{+}$gene was tagged with the c-Myc epitope at the C-terminus marked with the hph selection marker gene, which confers resistance to $100 \mu \mathrm{g} \mathrm{ml}^{-1}$ hygromycin $\mathrm{B}$. We then induced random point mutations into scm3-myc-hph DNA fragments through an error-prone PCR reaction. The mutated fragments were then introduced into the mis6GFP-kan strain to induce homologous recombination with the endogenous $\mathrm{scm} 3^{+}$gene. Colonies grown on YE5S + hygromycin $\mathrm{B}$ at $25^{\circ} \mathrm{C}$ were replicated onto YE5S plates containing $2 \mu \mathrm{g} \mathrm{ml}^{-1}$ phloxine $\mathrm{B}$ at $36^{\circ} \mathrm{C}$. Colonies showing temperature sensitivity with the normal localisation of Mis6-GFP were selected for sequencing and further experiments.

Chromatin IP Cells were cultured at $25^{\circ} \mathrm{C}$ in YE5S, followed by a temperature shift up 
to $36^{\circ} \mathrm{C}$ for $6 \mathrm{~h}$. The cells were then fixed with $1 \%$ formaldehyde for $10 \mathrm{~min}$ at $36^{\circ} \mathrm{C}$ and left on ice for $50 \mathrm{~min}$. Chromatin IP assays in this study were carried out as previously described ${ }^{11}$, with minor modifications. In brief, fixed cells were washed four times with Buffer I (50 mM HEPES/NaOH [pH 7.5], $140 \mathrm{mM} \mathrm{NaCl,} 1 \mathrm{mM}$ EDTA [pH7.5], 1\% Triton $\mathrm{X}-100$ and $0.1 \%$ sodium deoxycholate) at $4{ }^{\circ} \mathrm{C}$ and kept frozen at $-80^{\circ} \mathrm{C}$. Cells were then suspended in Buffer I supplemented with Complete Protease Inhibitor cocktail (Roche) and $1 \mathrm{mM}$ phenylmethylsulfonyl fluoride. Cells were destroyed using acid-washed glass beads and a FastPrep-24 bead shocker $(4 \times 20 \mathrm{sec}$, power $=6.0)$. The cell lysates were then sonicated using a sonifier VP-050 (PWM 10\%). A round of iterative sonication for $10 \mathrm{sec}$ comprising ON $(0.2 \mathrm{sec})$ and OFF $(0.4 \mathrm{sec})$ was repeated 10 or 15 times to shear chromatin DNA into fragments. Lysates were then centrifuged $(14,000 \mathrm{rpm}$ for $15 \mathrm{~min}$ at $4^{\circ} \mathrm{C}$ ) to collect supernatants, and the concentration was adjusted to $10 \mathrm{mg} \mathrm{ml}^{-1}$.

For immunoprecipitation, the rabbit anti-RNA polymerase II (phosphoS5) polyclonal antibody (1:100; ab5131) or rabbit anti-GFP polyclonal antibody (1:250; Clontech, 632592) was incubated with $200 \mu \mathrm{L}$ of the lysate for $1 \mathrm{~h}$ at $4^{\circ} \mathrm{C}$. Protein $\mathrm{A}$ sepharose (GE) was then added, incubated for $2 \mathrm{~h}$ at $4^{\circ} \mathrm{C}$ and washed three times with Buffer I, followed by suspension with Buffer I' (50 mM HEPES/NaOH [pH 7.5], $500 \mathrm{mM}$ $\mathrm{NaCl}, 1 \mathrm{mM}$ EDTA [pH 7.5], 1\% Triton $\mathrm{X}-100$ and $0.1 \%$ sodium deoxycholate) thrice, Buffer II (10 mM Tris-HCl [pH 8.0], $250 \mathrm{mM} \mathrm{LiCl,} \mathrm{0.5 \%} \mathrm{NP-40,} \mathrm{0.5 \%} \mathrm{sodium}$ deoxycholate) twice and TE twice. The amounts of DNA derived from whole-cell extracts and ChIP samples were assessed via quantitative PCR on the StepOne Real-Time PCR system (Applied Biosystems) using SYBR Green (TOYOBO). Oligonucleotide primers for detecting the central core region of centromeres were used as previously described ${ }^{61}$.

Microscopy Living cells were transferred to a glass-bottom dish (Matsunami) pre-coated with lectin, and the dish was filled with liquid EMM preheated at $25^{\circ} \mathrm{C}$ or $36^{\circ} \mathrm{C}$. Mounted cells were observed at $25^{\circ} \mathrm{C}$ or $36^{\circ} \mathrm{C}$ using a microscope (IX71, Olympus) with the DeltaVision-SoftWoRx system (Applied Precision), as previously described ${ }^{62}$. Cells were imaged in 12 sections at $0.4-\mu \mathrm{m}$ intervals along the z-axis. The temperature conditions are shown below. In Fig. 1, Cells were observed for $1 \mathrm{~h}$ after culturing at $36^{\circ} \mathrm{C}$ for $3 \mathrm{~h}$. In Fig. S1d, overnight cultures were incubated at $36^{\circ} \mathrm{C}$ for $4 \mathrm{~h}$, and cells were fixed with $3.2 \%$ formaldehyde (Thermo Fisher Scientific) for $20 \mathrm{~min}$. In Figs. 3c and S2, cells were cultured at $25^{\circ} \mathrm{C}$ for $20 \mathrm{~h}$ in EMM and imaged. The acquired images were processed as follows: images taken along the z-axis were deconvoluted and projected into a single image using the Quick Projection algorithm in the SoftWoRx software (v3.7.0 and v.6.5.1).

The fluorescence intensity of Cnp1 visualised with GFP or mCherry was 
measured using the Data Inspector command in SoftWoRx. The mean intensity of a single dot signal of Cnp1-GFP or GFP-Cnp1 in $6 \times 6$ pixels was measured, and the background signal outside of the nucleus was subtracted. For photobleaching of a Cnp1GFP dot signal, the dot area was successively irradiated four times by the $488 \mathrm{~nm}$ laser (50\% in power; Seki Technotron) for 0.05 sec using the SoftWoRx-QLM system. In Figs. $\mathbf{1}, \mathbf{2}, \mathbf{3 c}, \mathbf{4}, \mathbf{5}$ and S4, the resolution of enlarged images was adjusted from 72 to 144 pixels inch-1 using Adobe Photoshop (ver. 2021).

Drug treatment To inhibit RNAPII, 1,10-phenanthroline (Sigma-Aldrich) diluted to 100 $\mathrm{mg} \mathrm{ml}^{-1}$ with ethanol was added to the EMM at a final concentration of $100 \mu \mathrm{g} \mathrm{ml}^{-1}$. Alternatively, thiolutin (Wako) diluted to $5 \mathrm{mg} \mathrm{ml}^{-1}$ with DMSO was added to EMM at a final concentration of $20 \mu \mathrm{g} \mathrm{ml}^{-1}$ as previously described ${ }^{63}$. For mock treatment, the same amount of solvent (ethanol or DMSO) was added to the EMM. Observations began 15 min after the addition of reagents. To arrest cells at metaphase for more than $1 \mathrm{~h}$, the microtubule poison carbendazim (CBZ; Sigma-Aldrich) diluted to $5 \mathrm{mg} \mathrm{ml}^{-1}$ with DMSO was added to EMM at a final concentration of $50 \mu \mathrm{g} \mathrm{ml}^{-1}$ immediately before observation. To arrest cells during the G1/S phase, hydroxyurea (HU; Sigma-Aldrich) diluted to $1.5 \mathrm{M}$ with DMSO was added to YE5S at a final concentration of $15 \mathrm{mM}$.

\section{Chromosome segregation assay with cen2-GFP}

To evaluate chromosome segregation in cut9-665 and cut9-665 spt6 $\Delta$ mutants, the cen2-GFP system was employed to visualise the position of chromosome II centromere with GFP ${ }^{48}$. For metaphase arrest, cells were first arrested at the G1/S phase using $\mathrm{HU}$ for $2 \mathrm{~h}$. In the presence of $\mathrm{HU}$, the temperature was then shifted up to $36^{\circ} \mathrm{C}$ to inactivate Cut9 (an APC/C component). Cells were then washed with $\mathrm{ddH}_{2} \mathrm{O}$ three times and cultured in YE5S for $2 \mathrm{~h}$ at $36^{\circ} \mathrm{C}$ to release cells from $\mathrm{G} 1 / \mathrm{S}$ arrest until metaphase arrest via Cut9-inactivation. The culture was shifted down to $25^{\circ} \mathrm{C}$ again to finally release cells from metaphase to anaphase, and the distribution of cen2-GFP dots was observed for $1 \mathrm{~h}$. 


\section{References}

1. Walczak, C. E., Cai, S. \& Khodjakov, A. Mechanisms of chromosome behaviour during mitosis. Nat. Rev. Mol. Cell Biol. 11, 91-102 (2010).

2. Biggins, S. The composition, functions, and regulation of the budding yeast kinetochore. Genetics 194, 817-846 (2013).

3. Pidoux, A. L. \& Allshire, R. C. Kinetochore and heterochromatin domains of the fission yeast centromere. Chromosome Res. 12, 521-534 (2004).

4. Schueler, M. G. \& Sullivan, B. A. Structural and functional dynamics of human centromeric chromatin. Annu. Rev. Genomics Hum. Genet. 7, 301-313 (2006).

5. Holland, A. J. \& Cleveland, D. W. Boveri revisited: chromosomal instability, aneuploidy and tumorigenesis. Nat. Rev. Mol. Cell Biol. 10, 478-487 (2009).

6. Jansen, L. E. T., Black, B. E., Foltz, D. R. \& Cleveland, D. W. Propagation of centromeric chromatin requires exit from mitosis. J. Cell Biol. 176, 795-805 (2007).

7. Lando, D. et al. Quantitative single-molecule microscopy reveals that CENP-ACnp1 deposition occurs during G2 in fission yeast. Open Biol. 2, 120078 (2012).

8. Fujita, Y. et al. Priming of centromere for CENP-A recruitment by human hMis18a, hMis18ß, and M18BP1. Dev. Cell 12, 17-30 (2007).

9. Hayashi, T. et al. Mis16 and Mis18 are required for CENP-A loading and histone deacetylation at centromeres. Cell 118, 715-729 (2004).

10. Hayashi, T. et al. Schizosaccharomyces pombe centromere protein Mis19 links Mis16 and Mis18 to recruit CENP-A through interacting with NMD factors and the SWI/SNF complex. Genes Cells 19, 541-554 (2014).

11. Hirai, H., Arai, K., Kariyazono, R., Yamamoto, M. \& Sato, M. The kinetochore protein Kis1/Eic1/Mis19 ensures the integrity of mitotic spindles through maintenance of kinetochore factors Mis6/CENP-I and CENP-A. PLOS ONE 9, e111905 (2014).

12. Subramanian, L., Toda, N. R. T., Rappsilber, J. \& Allshire, R. C. Eic1 links Mis18 with the CCAN/Mis6/Ctf19 complex to promote CENP-A assembly. Open Biol. 4, 140043 (2014).

13. Foltz, D. R. et al. Centromere-specific assembly of CENP-a nucleosomes is mediated by HJURP. Cell 137, 472-484 (2009).

14. Dunleavy, E. M. et al. HJURP is a cell-cycle-dependent maintenance and deposition factor of CENP-A at centromeres. Cell 137, 485-497 (2009).

15. Williams, J. S., Hayashi, T., Yanagida, M. \& Russell, P. Fission yeast Scm3 mediates stable assembly of Cnp1/CENP-A into centromeric chromatin. Mol. Cell 
33, 287-298 (2009).

16. Pidoux, A. L. et al. Fission yeast Scm3: A CENP-A receptor required for integrity of subkinetochore chromatin. Mol. Cell 33, 299-311 (2009).

17. Dion, M. F. et al. Dynamics of replication-independent histone turnover in budding yeast. Science 315, 1405-1408 (2007).

18. Jin, C. et al. H3.3/H2A.Z double variant-containing nucleosomes mark 'nucleosome-free regions' of active promoters and other regulatory regions. Nat. Genet. 41, 941-945 (2009).

19. Deal, R. B., Henikoff, J. G. \& Henikoff, S. Genome-wide kinetics of nucleosome turnover determined by metabolic labeling of histones. Science 328, 1161-1164 (2010).

20. Smoak, E. M., Stein, P., Schultz, R. M., Lampson, M. A. \& Black, B. E. Long-Term Retention of CENP-A Nucleosomes in Mammalian Oocytes Underpins Transgenerational Inheritance of Centromere Identity. Curr. Biol. 26, 1110-1116 (2016).

21. Talbert, P. B. \& Henikoff, S. Transcribing Centromeres: Noncoding RNAs and Kinetochore Assembly. Trends in Genetics 34, 587-599 (2018).

22. Bergmann, J. H. et al. Epigenetic engineering: histone H3K9 acetylation is compatible with kinetochore structure and function. J. Cell. Sci. 125, 411-421 (2012).

23. Carlsten, J. O. et al. Mediator promotes CENP-A incorporation at fission yeast centromeres. Mol. Cell. Biol 32, 4035-4043 (2012).

24. Niikura, Y. et al. CENP-A K124 Ubiquitylation Is Required for CENP-A Deposition at the Centromere. Dev. Cell 32, 589-603 (2015).

25. Carroll, C. W., Silva, M. C. C., Godek, K. M., Jansen, L. E. T. \& Straight, A. F. Centromere assembly requires the direct recognition of CENP-A nucleosomes by CENP-N. Nat. Cell Biol. 11, 896-902 (2009).

26. Carroll, C. W., Milks, K. J. \& Straight, A. F. Dual recognition of CENP-A nucleosomes is required for centromere assembly. J. Cell Biol. 189, 1143-1155 (2010).

27. Falk, S. J. et al. CENP-C reshapes and stabilizes CENP-A nucleosomes at the centromere. Science 348, 699-703 (2015).

28. Guo, L. Y. et al. Centromeres are maintained by fastening CENP-A to DNA and directing an arginine anchor-dependent nucleosome transition. Nat. Commun. 8, 15775 (2017).

29. Cao, S., Zhou, K., Zhang, Z., Luger, K. \& Straight, A. F. Constitutive centromere- 
associated network contacts confer differential stability on CENP-A nucleosomes in vitro and in the cell. Mol. Biol. Cell 29, 751-762 (2018).

30. Zasadzińska, E. et al. Inheritance of CENP-A Nucleosomes during DNA Replication Requires HJURP. Dev. Cell 47, 348-362.e7 (2018).

31. Bobkov, G. O. M. et al. Spt6 is a maintenance factor for centromeric CENP-A. Nat. Commun. 11, 2919-14 (2020).

32. Takahashi, K., Chen, E. S. \& Yanagida, M. Requirement of Mis6 centromere connector for localizing a CENP-A-like protein in fission yeast. Science 288, 2215-2219 (2000).

33. Saitoh, S., Takahashi, K. \& Yanagida, M. Mis6, a fission yeast inner centromere protein, acts during G1/S and forms specialized chromatin required for equal segregation. Cell 90, 131-143 (1997).

34. Russell, P. \& Nurse, P. cdc25+ functions as an inducer in the mitotic control of fission yeast. Cell 45, 145-153 (1986).

35. Radcliffe, P., Hirata, D., Childs, D., Vardy, L. \& Toda, T. Identification of novel temperature-sensitive lethal alleles in essential $\beta$-tubulin and nonessential a2tubulin genes as fission yeast polarity mutants. Mol. Biol. Cell 9, 1757-1771 (1998).

36. Samejima, I. \& Yanagida, M. Bypassing anaphase by fission yeast cut9 mutation: requirement of cut9+ to initiate anaphase. J. Cell Biol. 127, 1655-1670 (1994).

37. Pidoux, A. L., Richardson, W. \& Allshire, R. C. Sim4: a novel fission yeast kinetochore protein required for centromeric silencing and chromosome segregation. J. Cell Biol. 161, 295-307 (2003).

38. Bahler, J. et al. Role of polo kinase and Mid1p in determining the site of cell division in fission yeast. J. Cell Biol. 143, 1603-1616 (1998).

39. Mulvihill, D. P., Petersen, J., Ohkura, H., Glover, D. M. \& Hagan, I. M. Plo1 kinase recruitment to the spindle pole body and its role in cell division in Schizosaccharomyces pombe. Mol. Biol. Cell 10, 2771-2785 (1999).

40. Takahashi, K., Yamada, H. \& Yanagida, M. Fission yeast minichromosome loss mutants mis cause lethal aneuploidy and replication abnormality. Mol. Biol. Cell 5, 1145-1158 (1994).

41. Nabetani, A., Koujin, T., Tsutsumi, C., Haraguchi, T. \& Hiraoka, Y. A conserved protein, Nuf2, is implicated in connecting the centromere to the spindle during chromosome segregation: a link between the kinetochore function and the spindle checkpoint. Chromosoma 110, 322-334 (2001).

42. Goshima, G., Saitoh, S. \& Yanagida, M. Proper metaphase spindle length is 
determined by centromere proteins Mis12 and Mis6 required for faithful chromosome segregation. Genes Dev. 13, 1664-1677 (1999).

43. Rothbauer, U. et al. Targeting and tracing antigens in live cells with fluorescent nanobodies. Nat. Methods 3, 887-889 (2006).

44. Avvakumov, N., Nourani, A. \& Côté, J. Histone Chaperones: Modulators of Chromatin Marks. Mol. Cell 41, 502-514 (2011).

45. Hammond, C. M., Strømme, C. B., Huang, H., Patel, D. J. \& Groth, A. Histone chaperone networks shaping chromatin function. Nat. Rev. Mol. Cell Biol. 18, 141-158 (2017).

46. Lee, J. et al. Chromatin remodeller Fun30 Fft3 induces nucleosome disassembly to facilitate RNA polymerase II elongation. Nat. Commun. 8, 14527 (2017).

47. Sadeghi, L., Siggens, L., Svensson, J. P. \& Ekwall, K. Centromeric histone H2B monoubiquitination promotes noncoding transcription and chromatin integrity. Nat. Struct. Mol. Biol. 21, 236-243 (2014).

48. Yamamoto, A. \& Hiraoka, Y. Monopolar spindle attachment of sister chromatids is ensured by two distinct mechanisms at the first meiotic division in fission yeast. EMBO J. 22, 2284-2296 (2003).

49. Yoh, S. M., Cho, H., Pickle, L., Evans, R. M. \& Jones, K. A. The Spt6 SH2 domain binds Ser2-P RNAPII to direct Iws1-dependent mRNA splicing and export. Genes Dev. 21, 160-174 (2007).

50. Gonzalez, M., He, H., Sun, S., Li, C. \& Li, F. Cell cycle-dependent deposition of CENP-A requires the Dos1/2-Cdc20 complex. Proc. Natl. Acad. Sci. U.S.A. 110, 606-611 (2013).

51. Stankovic, A. et al. A Dual Inhibitory Mechanism Sufficient to Maintain Cell-CycleRestricted CENP-A Assembly. Mol. Cell 65, 231-246 (2017).

52. Swaffer, M. P., Jones, A. W., Flynn, H. R., Snijders, A. P. \& Nurse, P. CDK Substrate Phosphorylation and Ordering the Cell Cycle. Cell 167, 1750-1761.e16 (2016).

53. Nishihashi, A. et al. CENP-I is essential for centromere function in vertebrate cells. Dev. Cell 2, 463-476 (2002).

54. Okada, M. et al. The CENP-H-I complex is required for the efficient incorporation of newly synthesized CENP-A into centromeres. Nat. Cell Biol. 8, 446-457 (2006).

55. Moreno, S., Klar, A. \& Nurse, P. Molecular genetic analysis of fission yeast Schizosaccharomyces pombe. Meth. Enzymol. 194, 795-823 (1991).

56. Bahler, J. et al. Heterologous modules for efficient and versatile PCR-based gene targeting in Schizosaccharomyces pombe. Yeast 14, 943-951 (1998). 
57. Sato, M., Dhut, S. \& Toda, T. New drug-resistant cassettes for gene disruption and epitope tagging in Schizosaccharomyces pombe. Yeast 22, 583-591 (2005).

58. Tanaka, K., Chang, H. L., Kagami, A. \& Watanabe, Y. CENP-C functions as a scaffold for effectors with essential kinetochore functions in mitosis and meiosis. Dev. Cell 17, 334-343 (2009).

59. Takayama, Y. et al. Biphasic incorporation of centromeric histone CENP-A in fission yeast. Mol. Biol. Cell 19, 682-690 (2008).

60. Kakui, Y. et al. Module-based construction of plasmids for chromosomal integration of the fission yeast Schizosaccharomyces pombe. Open Biol. 5, (2015).

61. Yokobayashi, S., Yamamoto, M. \& Watanabe, Y. Cohesins determine the attachment manner of kinetochores to spindle microtubules at meiosis I in fission yeast. Mol. Cell. Biol 23, 3965-3973 (2003).

62. Sato, M., Toya, M. \& Toda, T. Visualization of fluorescence-tagged proteins in fission yeast: the analysis of mitotic spindle dynamics using GFP-tubulin under the native promoter. Methods Mol. Biol. 545, 185-203 (2009).

63. Takemata, N. et al. Local potentiation of stress-responsive genes by upstream noncoding transcription. Nucleic Acids Res. 44, 5174-5189 (2016). 
a

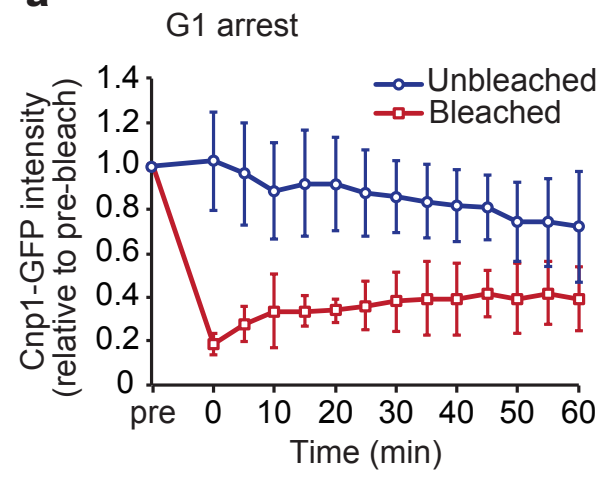

G2 arrest

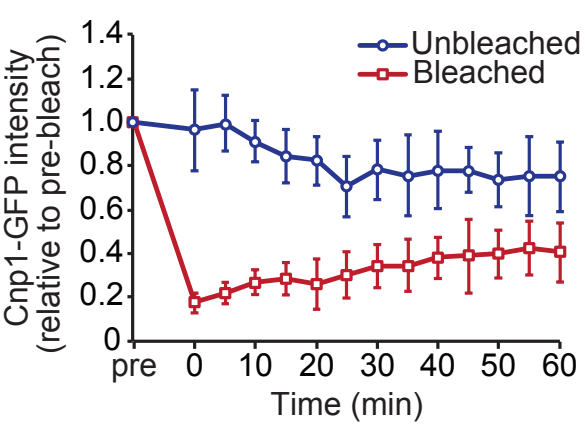

e

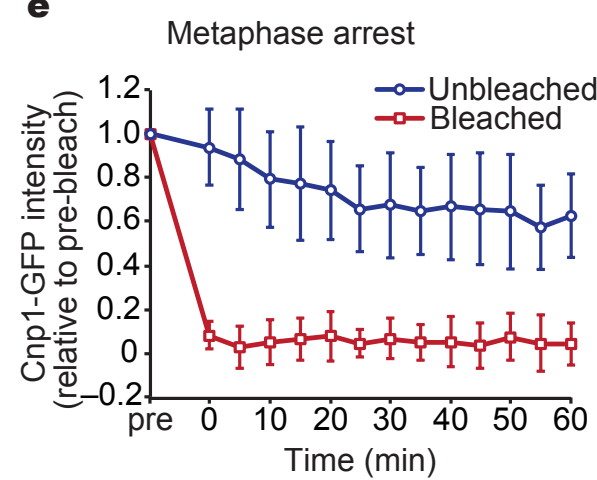

b

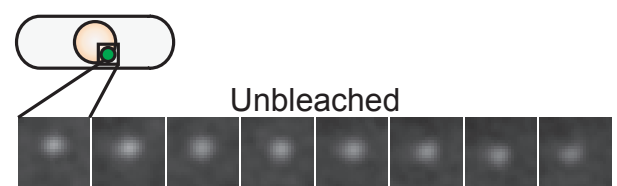

Bleached

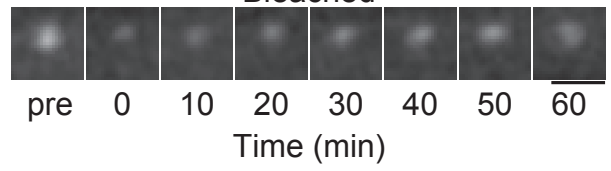

d

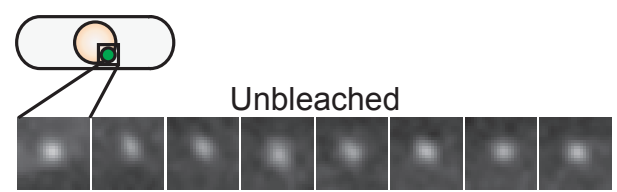

Bleached

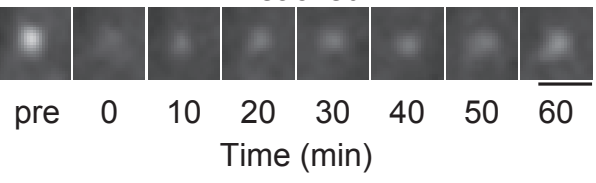

f

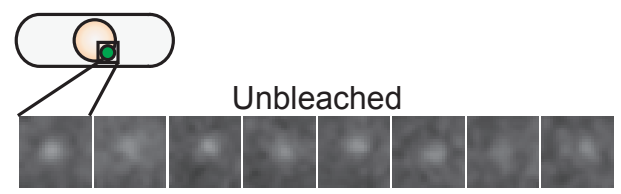

Bleached

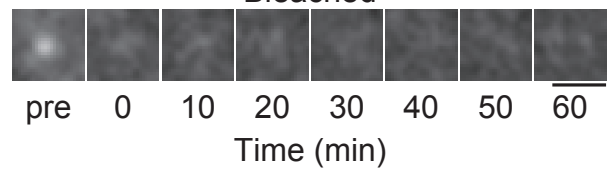

Fig. 1 Deposition of Cnp1 (CENP-A) ceases in metaphase

Fluorescence recovery after photobleaching (FRAP) assays were performed with Cnp1GFP cells arrested at each stage of the cell cycle. Cells with unbleached centromeres were used as controls. (a,c,e) Recovery kinetics of Cnp1-GFP fluorescence over time. Intensities are shown normalised to values before bleaching (pre). (b,d,f) Time-lapse Cnp1-GFP images of representative cells. Cells were arrested in G1 (using the cdc10 mutant; a,b), G2 (cdc25; c,d) or metaphase (alp12; e,f), and Cnp1-GFP dots were photobleached. Fluorescence recovery at the centromeres was then monitored over time. Numbers of unbleached and bleached cells: $n=11$ and 16 (a); $n=9$ and 9 (c); $n=19$ and 15 (e), respectively. Data are presented as the mean \pm s.d. Scale bars $=2 \mu \mathrm{m}$. 
a

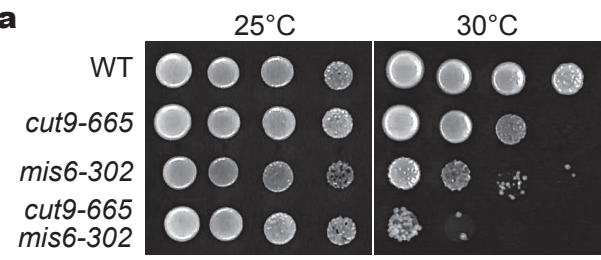

c

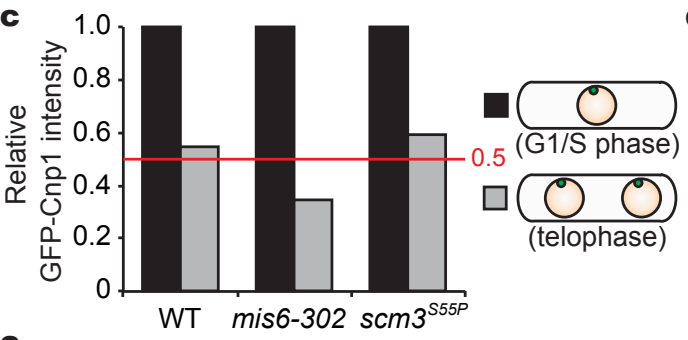

e

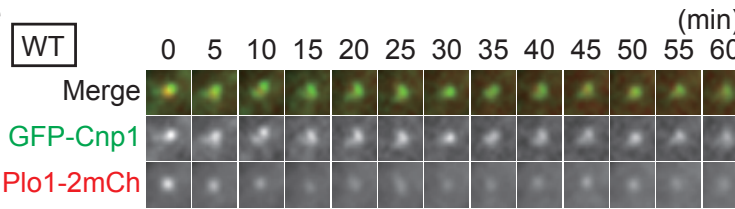

mis6-302

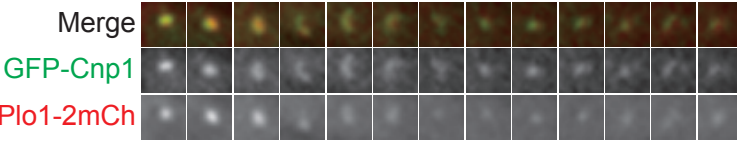

g

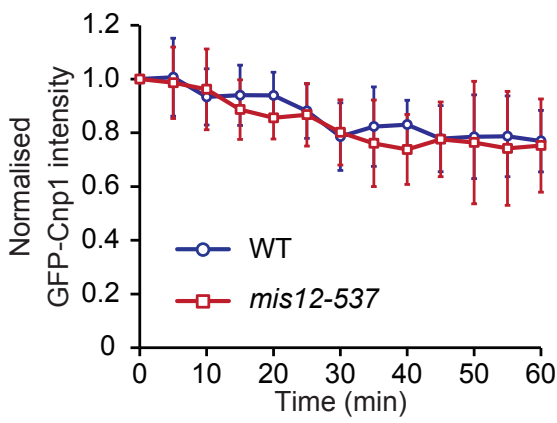

b

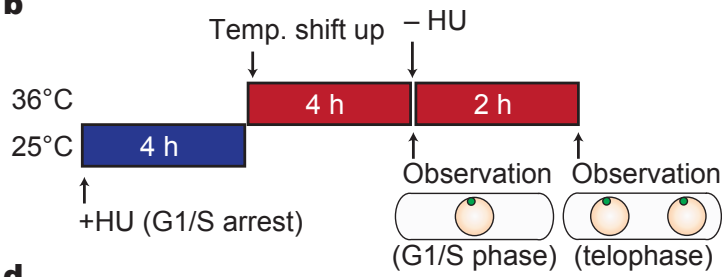

d

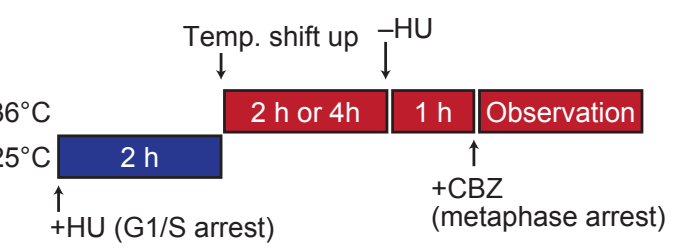

Fig. 2 Mis6 is required for Cnp1 maintenance at the centromere during metaphase

(a) Growth assays for indicated strains. Cells at 10-fold serial dilutions were grown at 25 and $30^{\circ} \mathrm{C}$. (b,c) Comparison of GFP-Cnp1 signals before and after mitosis. Experimental design (b). Cells were synchronised at $\mathrm{G} 1 / \mathrm{S}$ using hydroxyurea $(\mathrm{HU})$ at $25^{\circ} \mathrm{C}$, followed by a shift up to the restrictive temperature $\left(36^{\circ} \mathrm{C}\right)$ for mis6-302 and $\mathrm{scm} 3^{\mathrm{S} 55 P}$ mutants in advance. The cells were then released to late mitosis via HU removal. GFP-Cnp1 fluorescence intensity was measured before (G1/S) and after release from HU (telophase). GFP-Cnp1 intensities in WT, mis6-302 and scm3 ${ }^{555 P}$ cells were quantified ( $n \geq 22$ cells, c). The data is normalised as a ratio of values for telophase to interphase. (d-h) Cnp1 maintenance assays using GFP-Cnp1 Plo1-2mCherry cells of the WT, mis6302, mis12-537 and nuf2-2 background. (d) Experimental design. Cells were synchronised at $\mathrm{G} 1 / \mathrm{S}$ via $\mathrm{HU}$ treatment at $25^{\circ} \mathrm{C}$ and shifted up to $36^{\circ} \mathrm{C}$ for $2 \mathrm{~h}$ to inactivate Mis6 and Alp12 or for $4 \mathrm{~h}$ to inactivate Mis12, Nuf2 and Alp12 in advance. Cells were released from HU and arrested in mitosis using CBZ. (e) Time-lapse images of the GFP-Cnp1 signal (green) during metaphase in WT and mis6-302 cells. Plo1-2mCh 
(red) is shown as a mitotic marker (Scale bar: $2 \mu \mathrm{m})$. (f-h) Temporal kinetics of GFPCnp1 intensities during metaphase arrest, normalised to values for 0 min. (f) WT, $n=16$ cells; mis6-302, $n=20$ cells. Data are presented as the mean \pm s.d. (g) WT, $n=11$ cells; mis 12-537, $n=6$ cells. Data are presented as the mean \pm s.d. (h) WT, $n=21$ cells; nuf22, $n=18$ cells. Data are presented as the mean \pm s.d. 
a

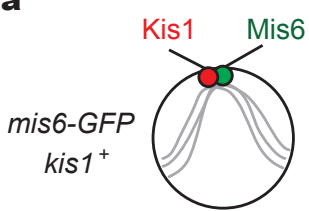

Interphase

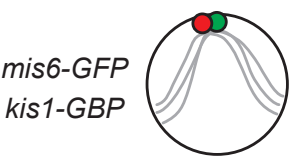

c

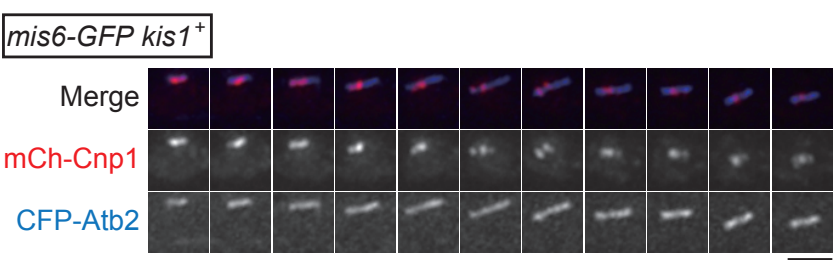

mis6-GFP kis1-GBP

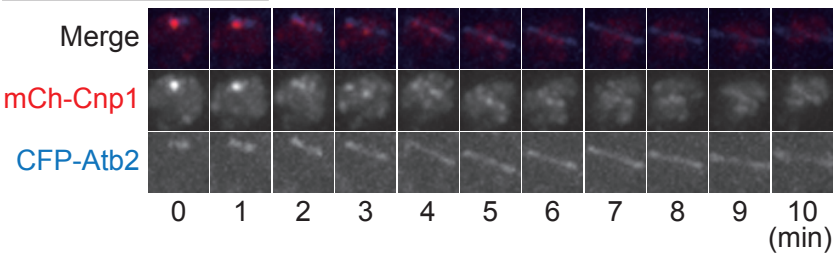

Metaphase
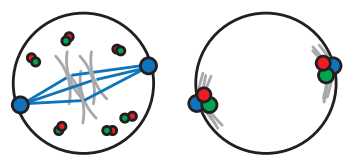

d

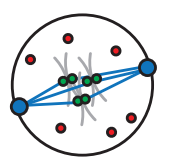

CFP-Atb

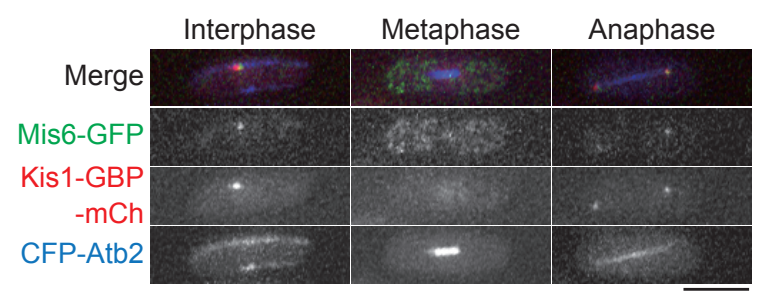

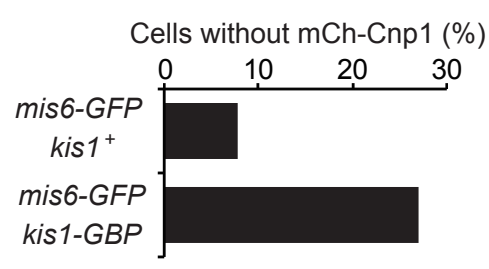

Fig. 3 Metaphase-specific removal of Mis6 causes a decrease of Cnp1

(a) Schematics illustrating the outline of knocksideways to remove Mis6 from centromeres only during metaphase using Kis1. Kis1 (red) and Mis6 (green) co-localise to centromeres during interphase, but Kis1 disperses at mitosis onset (top). In cells coexpressing Kis1-GBP and Mis6-GFP, Kis1-GBP brings Mis6-GFP out of centromeres only during metaphase (bottom). (b) Cells expressing Mis6-GFP (green), Kis1-GBPmCherry (red) and CFP-Atb2 (cyan fluorescent protein fused with a2-tubulin, blue) at each cell cycle stage. Scale bar $=5 \mu \mathrm{m}$. (c) Pro $\sim$ metaphase cells expressing Mis6-GFP, mCh-Cnp1 (red) and CFP-Atb2 (microtubules; cyan) together with untagged Kis1 (top) or Kis1-GBP (bottom) were imaged. Scale bar $=2 \mu \mathrm{m}$. (d) Population of metaphase cells with or without mCherry-Cnp1 at centromeres ( $n>50$ cells). (e) Growth assays for indicated strains. Cells at 10 -fold serial dilutions were grown in EMM at $36^{\circ} \mathrm{C}$. 
a
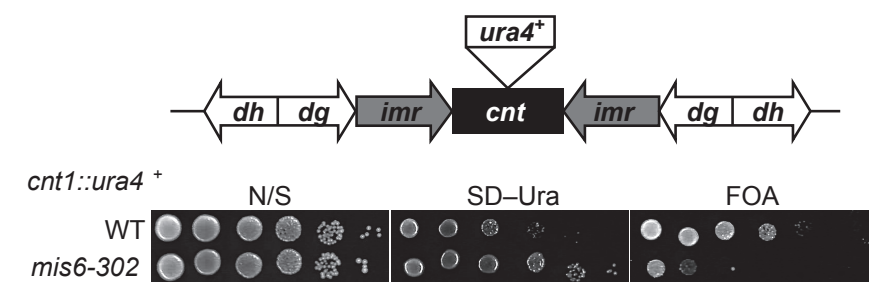

c
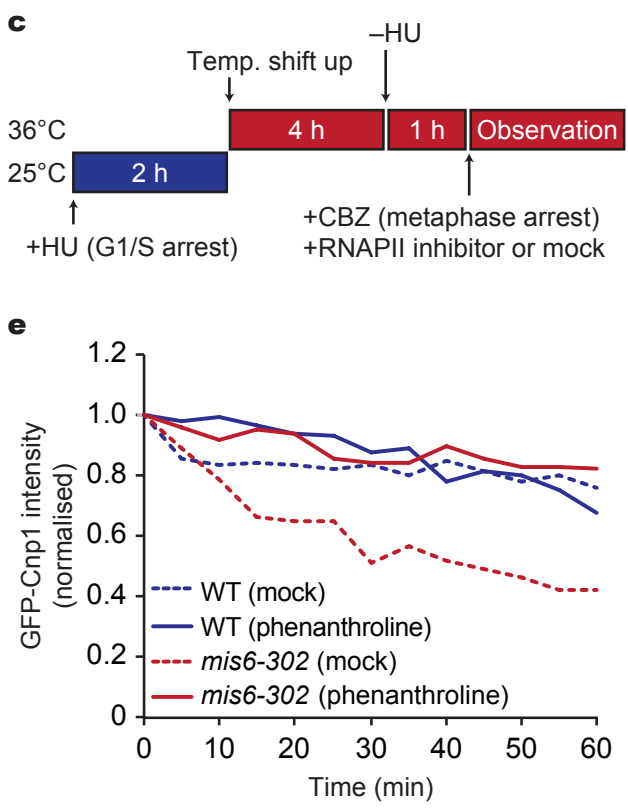

d
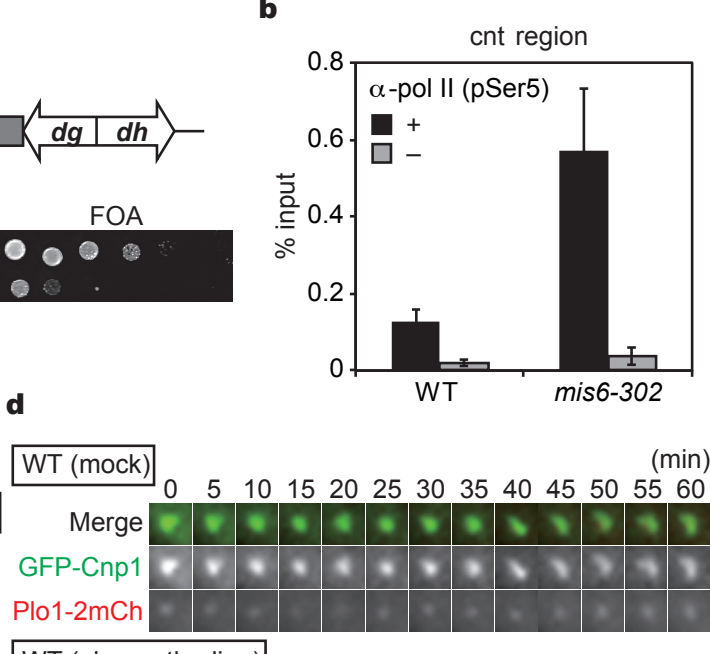

WT (phenanthroline)

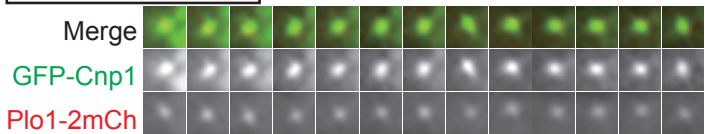

mis6-302 (mock)

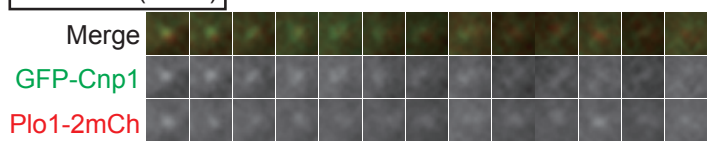

mis6-302 (phenanthroline)

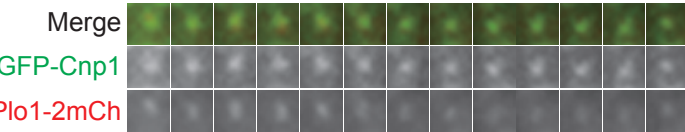

Fig. 4 Loss of Cnp1 in mis6-302 cells is coupled with centromeric transcription by RNA polymerase II

(a) A schematic illustrating the centromeric DNA structure (top). For silencing assays, the $\mathrm{ura4}^{+}$gene is inserted in the central core (cnt) region (cnt::ura4+) in chromosome I. $i m r$, the innermost repeats; $d g$ and $d h$ : the outer repeats. Silencing assays (bottom). 10fold serial dilution of WT and mis6-302 cells with cnt::ura4+, grown on nonselective (N/S), uracil-deficient (SD-Ura) and counter-selective (FOA) media at $25^{\circ} \mathrm{C}$. (b) Chromatin IP (ChIP) for phosphorylated RNA polymerase II (RNAPII pSer5) at cnt region (the central core region) in WT and mis6-302 cells. ChIP was performed with (+) or without $(-)$ the anti-pol II (pSer5) antibody. Error bars $= \pm \mathrm{s}$. d. ( $N=2$ experiments). (c-e) RNAPII was inhibited in WT and mis6-302 cells expressing GFP-Cnp1 and Plo1-2mCherry. (c) Experimental design. Cells arrested to $\mathrm{G} 1 / \mathrm{S}$ at $25^{\circ} \mathrm{C}$ were shifted up to $36^{\circ} \mathrm{C}$ to inactivate Mis6 or Alp12. The cells were then released into mitosis and arrested at metaphase using CBZ. Cells were treated with an RNAPII inhibitor (1,10-phenanthroline) or mock treatment prior to observation. (d) Time-lapse imaging of GFP-Cnp1 (green) with Plo1$2 \mathrm{mCh}$ (red) in WT and mis6-302 cells with or without the inhibitor. Scale bar $=2 \mu \mathrm{m}$. (e) A Cnp1 maintenance assay. Temporal kinetics of the GFP-Cnp1 fluorescence intensity during metaphase in the indicated samples were monitored. WT (mock), $n=22$ cells; mis6-302 (mock), $n=17$ cells; WT (phenanthroline), $n=30$ cells; mis6-302 (phenanthroline), $n=20$ cells. The data are normalised to the intensities at $0 \mathrm{~min}$, and the mean is shown. 
a

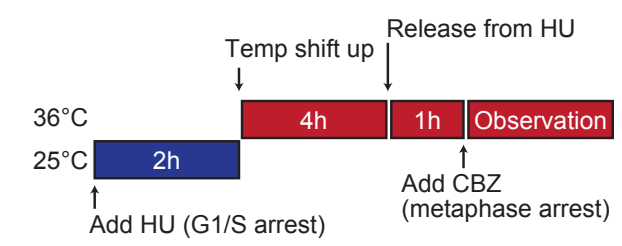

b

e
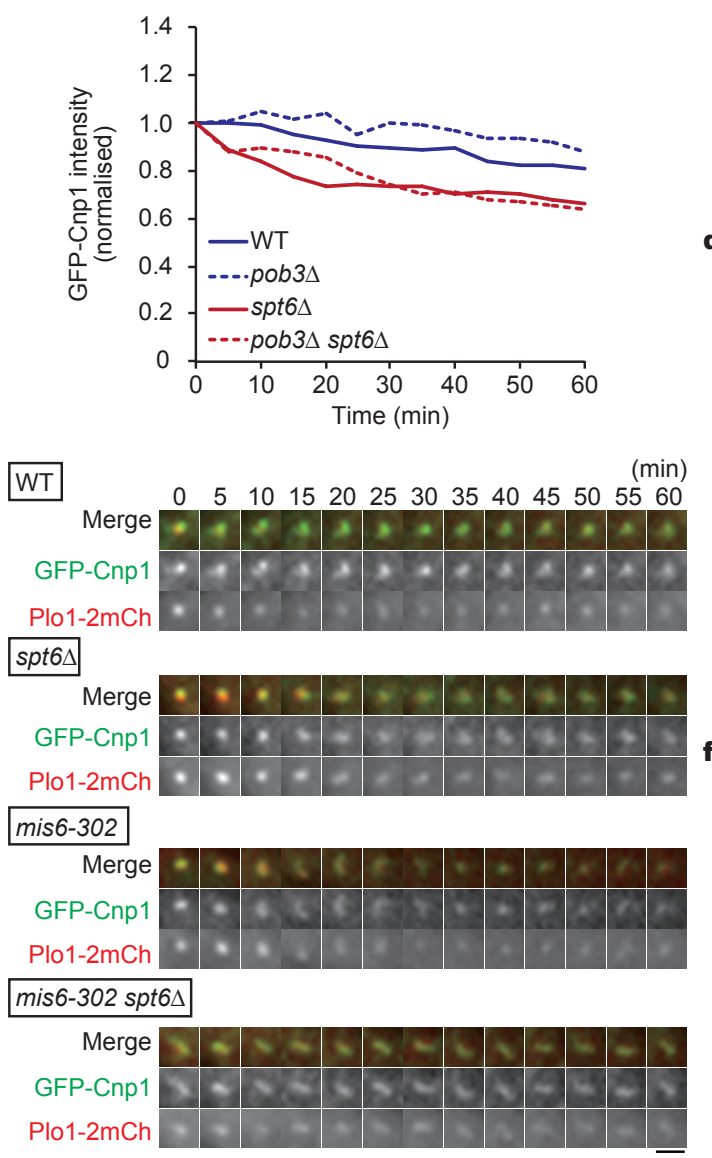

c

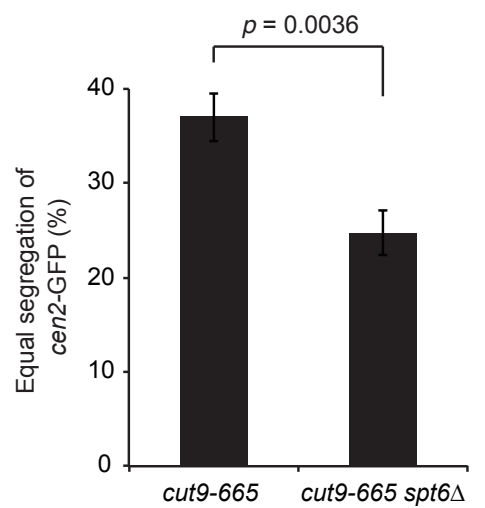

d
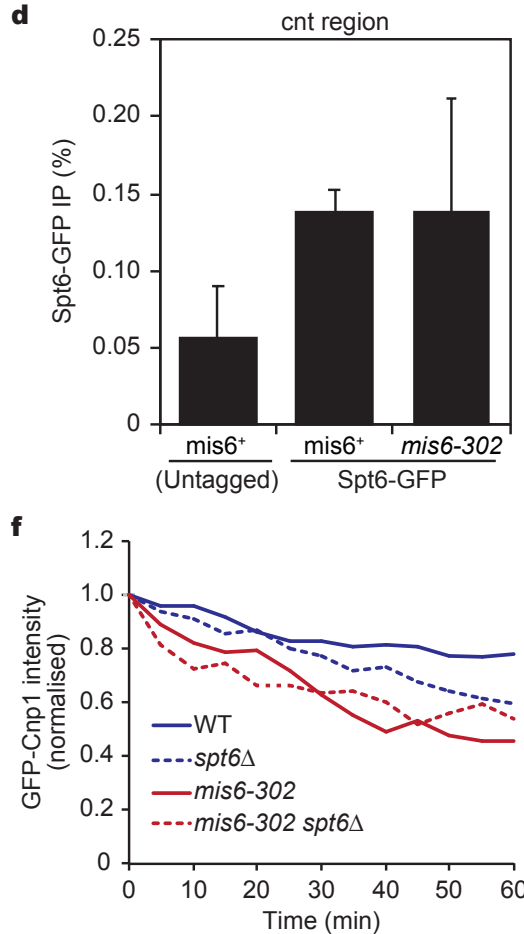

Fig. 5 Recycling of Cnp1 requires the chromatin remodelling factor Spt6

(a, b) Cnp1 maintenance assays during metaphase were performed using mutants of indicated chromatin remodellers. (a) Experimental design. Cells arrested to $\mathrm{G} 1 / \mathrm{S}$ at $25^{\circ} \mathrm{C}$ were shifted up to $36^{\circ} \mathrm{C}$ to inactivate Alp12. Cells were then released into mitosis and arrested at metaphase via CBZ treatment prior to observation. (b) A Cnp1 maintenance assay. Temporal kinetics of GFP-Cnp1 fluorescence intensity in metaphase were monitored for each mutant. WT, $n=18$ cells; pob3 $\Delta, n=14$ cells; spt6 $\Delta, n=16$ cells; pob3 $\Delta$ spt6 $\Delta, n=35$ cells. The data are normalised to intensities at $0 \mathrm{~min}$, and the mean is shown. (c) Population of cells with equal segregation of cen2-GFP (chromosome II centromere was visualised with GFP). Error bars $= \pm \mathrm{s}$. d., $N=3$ experiments. $p$ : Student's t-test (two-tailed). (d) ChIP of Spt6-GFP using at the cnt region in mis6 $6^{+}$and mis6-302 cells expressing Spt6-GFP or untagged Spt6. ChIP was performed with the anti-GFP antibody. Error bars $= \pm \mathrm{s}$. d., $N=3$ experiments. (e, f) Cnp1 maintenance assay. (e) Time-lapse images for cells expressing GFP-Cnp1 (green) and Plo1$2 \mathrm{mCherry} \mathrm{(red)} \mathrm{for} \mathrm{each} \mathrm{mutant} \mathrm{background.} \mathrm{Scale} \mathrm{bar}=2 \mu \mathrm{m}$. (f) WT, $n=16$ cells; mis6-302, $n=20$ cells; spt6 $\Delta, n=14$ cells; mis6-302 spt6 $\Delta, n=11$ cells. 